\title{
Unsupervised Perceptual Segmentation of Natural Color Images Using Fuzzy-Based Hierarchical Algorithm
}

\author{
Junji Maeda, Akimitsu Kawano, Sato Saga, and Yukinori Suzuki \\ Muroran Institute of Technology, Muroran 050-8585, Japan \\ junji@csse.muroran-it.ac.jp
}

\begin{abstract}
This paper proposes unsupervised perceptual segmentation of natural color images using a fuzzy-based hierarchical algorithm. $L^{\star} a^{\star} b^{\star}$ color space is used to represent color features and statistical geometrical features are adopted as texture features. A fuzzy-based homogeneity measure makes a fusion of color features and texture features. Proposed hierarchical segmentation method is performed in four stages: simple splitting, local merging, global merging and boundary refinement. Experiments on segmentation of natural color images are presented to verify the effectiveness of the proposed method in obtaining perceptual segmentation.
\end{abstract}

\section{Introduction}

Image segmentation is a process to partition an image into meaningful regions and is an important step before an image recognition process. In this paper, we are concerned with unsupervised perceptual segmentation of natural color images. Perceptual segmentation or rough segmentation is defined to obtain segmentation that produces a small number of segmented regions, and each region should represent a main object or a meaningful part of an object without paying much attention to region interiors. For example, perceptual segmentation could regard a tree that has many branches and leaves as one object in contrast to the conventional detailed segmentation.

Since natural color images contain various textures with different properties and all kinds of man-made objects, perceptual segmentation of natural color scenes is still a significantly difficult problem. Therefore, an effective segmentation method based on a set of texture features having good discriminating capability is essential in order to segment natural color images.

Though there is an extensive literature on image segmentation [1]-4, the papers on perceptual segmentation are limited. Among them, Mirmehdi and Petrou [5] proposed the method based on the multiscale perceptual tower and the probabilistic relaxization method. Shi and Malik [6] proposed the perceptual grouping method based on graph theory, and Ma and Manjunath [7] proposed the technique based on Gabor filter and edge flow. Recently, Chen et al. 8 proposed the approach based on the adaptive clustering algorithm and steerable 
filter decomposition. Although these methods perform well, their algorithms are complicated and difficult to implement.

Searching for simpler algorithm, we proposed to use local fractal dimension and a fuzzy region-growing algorithm for rough segmentation [9]-11]. However, our approach were not able to reduce the number of segmented regions sufficiently.

Ojala and Pietikäinen 12 proposed a texture segmentation method that has the advantages of a simple algorithm and easy implementation. Their method is a hierarchical segmentation algorithm that uses local binary pattern and contrast $(L B P / C)$ features as texture measures and executes segmentation in hierarchical splitting, agglomerative merging and pixelwise classification. Though the method performs well for perceptually uniform segmentation of texture images, it is insufficient to segment natural color images for the following reasons.

1) It is difficult to use a minimum block size smaller than 16 due to the unstable histogram distribution of $L B P / C$ features. Since the method is split-and-merge technique, it is preferable to use smaller block size at the split stage.

2) The method only treats gray-scale images and has to be adapted to color images.

3) The algorithm has a heavy computational burden due to the agglomerative merging stage since all possible pairs must be searched at each step just to merge two adjacent regions.

In this paper we propose a new technique, a fuzzy-based hierarchical algorithm, to perform unsupervised perceptual segmentation of natural color images based on the method by Ojala and Pietikäinen. We improve their algorithm in the following four points.

1) We adopt Statistical Geometrical Features (SGF) [13] as texture measures because it enables the algorithm to set a small minimum block size of 4 and the SGF can discriminate various types of textures remarkably.

2) We adopt fuzzy reasoning [14] to incorporate color features as well as texture features that enables the algorithm to treat color images.

3) We change the stage of hierarchical splitting into simple splitting to reduce the computational cost and the number of parameters.

4) We introduce a new stage of local merging that merges adjacent regions locally in order to drastically reduce the number of regions to be used at the stage of global merging. It is expected that the new stage will considerably reduce the total computational cost.

As a result of these improvement, the proposed algorithm has the capability of unsupervised perceptual segmentation of natural color images by the simple algorithm with easy implementation that has four hierarchical stages: simple splitting, local merging, global merging and boundary refinement. During the latter three stages, we measure the similarity of any adjacent regions by fuzzy homogeneity which combines the similarity of color features and texture features 
with different weights of importance. We use the $L^{\star} a^{\star} b^{\star}$ color space to represent color features and the SGF as texture features.

The adoption of fuzzy-based homogeneity measure simplifies the complex mechanism of integrating different features by using symbolic representations. It also reduces the difficulty in choosing the many threshold values inherent in segmentation methods, though the tuning of the fuzzy membership functions is still required for each image.

In this paper we also propose to introduce a new type of parameter that is the number of segmented regions $N$ instead of the threshold value. Since the expected number of segmented regions is very small in perceptual segmentation, it is possible for a user to presuppose a rough estimate of a desirable number of segmented regions in advance depending on the contents of each image and user's intention. We consider around 5-15 number of segmented regions as optimal range of $N$ in the sense of perceptual segmentation. In the practical implementation, the proposed algorithm has the significant ability to produce the segmentation results by reducing the number of segmented regions one by one at each step. Thus, the user is able to determine the optimal result with an appropriate roughness by observing the several segmented results.

Several experiments are made to confirm the effectiveness of the proposed method in obtaining unsupervised perceptual segmentation of natural color images.

\section{Color and Texture Features}

\section{$2.1 \quad L^{\star} a^{\star} b^{\star}$ Color Features}

The $L^{\star} a^{\star} b^{\star}$ color space is a perceptually uniform color space, where $L^{\star}$ represents brightness and $a^{\star}$ and $b^{\star}$ represent chromatic information. We obtain the $L^{\star} a^{\star} b^{\star}$ color space from the $R G B$ color space, then the three components are normalized and used as three color features.

\subsection{SGF Texture Features}

The SGF 13 are a set of texture features based on the statistics of geometrical properties of connected regions in a sequence of binary images obtained from an original image. The extraction of the SGF starts by thresholding the each component of a color image $C(x, y)$ (where $C=L^{\star}, a^{\star}$ and $b^{\star}$ ) with a threshold value $\alpha$ that produces the binary image $C_{b}$ defined as

$$
C_{b}(x, y ; \alpha)=\left\{\begin{array}{cc}
1 & C(x, y) \geq \alpha \\
0 & \text { otherwise }
\end{array}\right.
$$

where $1 \leq \alpha \leq n_{l}-1$ and $n_{l}$ is the maximum gray level of each component.

Each binary image $C_{b}(x, y ; \alpha)$ comprises a several connected regions. The number of connected regions of 1 -valued pixels and that of 0 -valued pixels give two geometrical measures, $N O C_{1}(\alpha)$ and $N O C_{0}(\alpha)$, respectively. Next a measure of irregularity (or a measure of non-circularity) is defined to each of the 


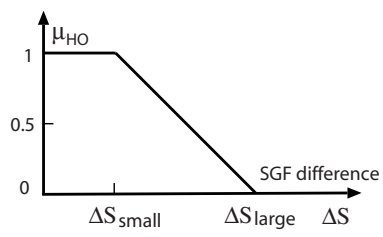

(a)

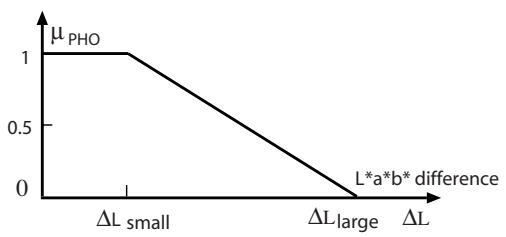

(b)

Fig. 1. Fuzzy membership functions of (a) SGF difference $(\Delta S)$ and (b) $L^{\star} a^{\star} b^{\star}$ difference $(\Delta L)$

connected regions. The average of irregularity measure of 1-valued pixels in the

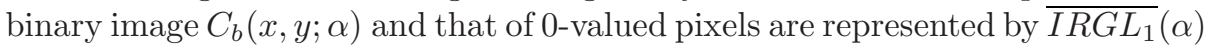
and $\overline{I R G L_{0}}(\alpha)$, respectively.

Each of these four functions is further characterized using the three statistics over an entire image: the average value, the sample mean and the sample standard deviation. This gives a total of 36 texture features. At the implementation, a sliding overlapping window of size $5 \times 5$ is used to calculate the SGF of each pixel of an original image.

\section{$3 \quad$ Fuzzy-Based Homogeneity Measure}

Homogeneity is a measure to test similarity of two regions under consideration during segmentation procedure. We adopt a fuzzy-based homogeneity measure to integrate the different features: the $L^{\star} a^{\star} b^{\star}$ color features and the SGF texture features. We use the following fuzzy rules where each rule has a corresponding membership function.

1) Rule 1: if SGF difference is SMALL, then HOMOGENEOUSE (HO); else NOT HOMOGENEOUSE (NHO).

2) Rule 2: if $L^{\star} a^{\star} b^{\star}$ difference is SMALL, then PROBABLY HOMOGENEOUSE (PHO); else PROBABLY NOT HOMOGENEOUSE (PNHO).

These fuzzy rules give the SGF texture features a higher priority than the $L^{\star} a^{\star} b^{\star}$ color features because we consider that the texture features provide more important information for textured color images. In these fuzzy rules, a SGF difference is the Euclidean distance of 36 SGF between two regions under consideration, and a $L^{\star} a^{\star} b^{\star}$ difference is the Euclidean distance of the three $L^{\star} a^{\star} b^{\star}$ components between them. Four conditions HO, NHO, PHO and PNHO represent different grades of homogeneity between two regions. Their homogeneity values $\mu_{H O}, \mu_{N H O}\left(=1-\mu_{H O}\right), \mu_{P H O}$ and $\mu_{P N H O}\left(=1-\mu_{P H O}\right)$ can be obtained from fuzzy membership functions of the SGF difference and the $L^{\star} a^{\star} b^{\star}$ difference as shown in Fig. 1. Here $\Delta S$ represents the SGF difference and $\Delta L$ represents the $L^{\star} a^{\star} b^{\star}$ difference. The values of $\Delta S_{\text {small }}, \Delta S_{\text {large }}, \Delta L_{\text {small }}$ and $\Delta L_{\text {large }}$ in Fig. 1 have to be tuned empirically.

A final homogeneity measure $H$ is inferred by min-max inference by using the fuzzy set as shown in Fig. 2 and the centroid defuzzification method 14. Suppose 


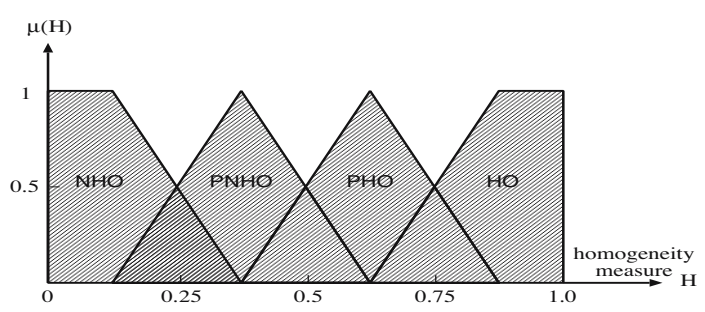

Fig. 2. The fuzzy set used for homogeneity inference

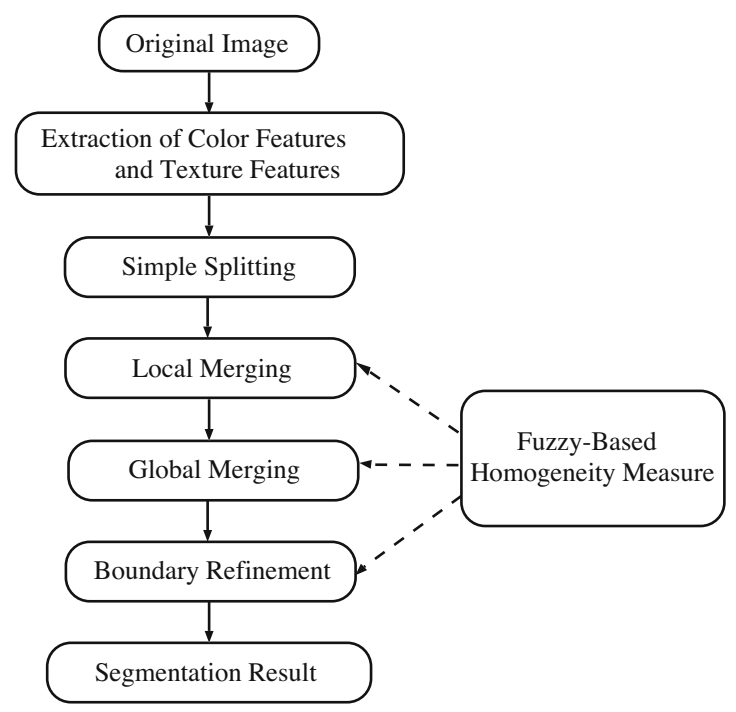

Fig. 3. Block diagram of the segmentation procedure

the homogeneity limit is set 0.5 , then if the inferred homogeneity measure is over 0.5, the two regions being concerned are regarded as homogeneous and they are merged. We use the value of the homogeneity measure $H$ in the proposed segmentation algorithm.

\section{Segmentation Algorithm}

The proposed fuzzy-based hierarchical segmentation procedure is shown in Fig. 3 . We first obtain the $L^{\star} a^{\star} b^{\star}$ color features and the SGF texture features for each pixel of an original image. We then execute the segmentation in four stages: simple splitting, local merging, global merging and boundary refinement. During the latter three stages, the fuzzy-based homogeneity measure $H$ is used as similarity measure. In the following, we will demonstrate the progress of segmentation on a $256 \times 256$ natural color image as shown in Fig. 4(a). 


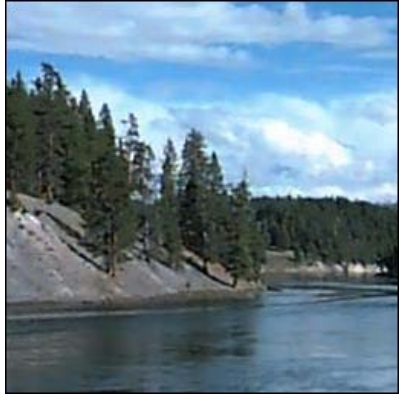

(a)

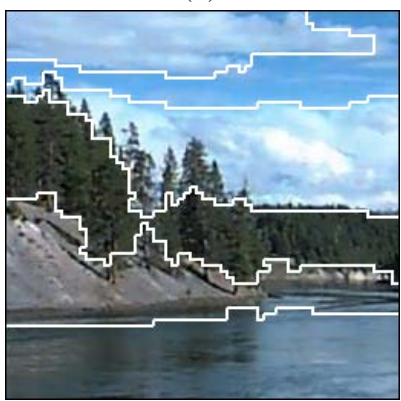

(d)

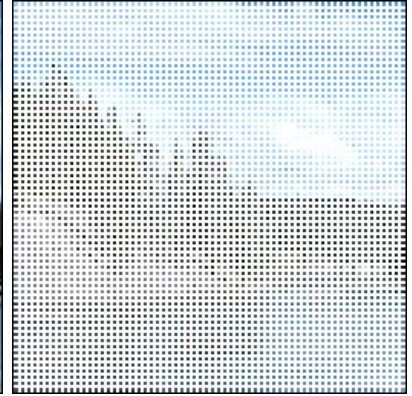

(b)

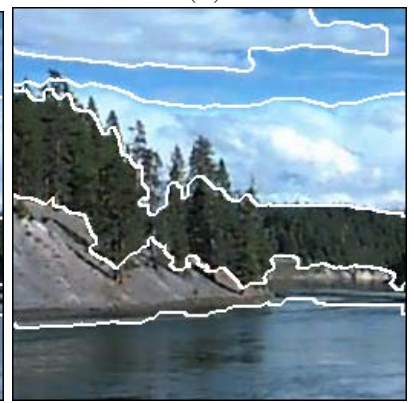

(e)

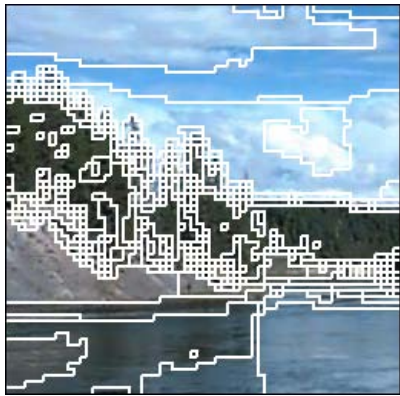

(c)

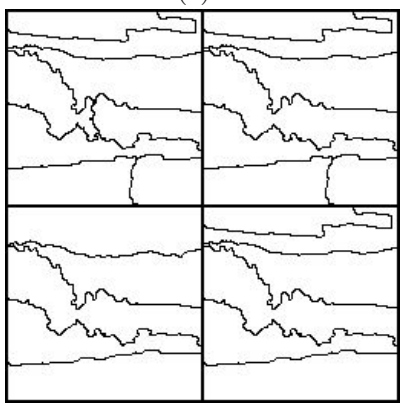

(f)

Fig. 4. Segmentation of a natural color image: (a) original image; (b) result of simple splitting; (c) result of local merging; (d) result of global merging with $N=6$; (e) result of boundary refinement; (f) results of segmentation with $N=8,7,6$ and 5 shown clockwise from the upper left

\subsection{Simple Splitting}

Ojala and Pietikäinen used the hierarchical splitting algorithm that recursively splits an original image into square blocks of varying size. Since their algorithm requires computational cost and the threshold value, we changed the stage of hierarchical splitting into simple splitting to reduce the computational cost and the number of parameters. In simple splitting, the image is divided into rectangular subblocks of size $4 \times 4$ as shown in Fig. $4(b)$. It is noted the adoption of the SGF texture features and the incorporation of local merging in our algorithm enable the use of simple splitting.

\subsection{Local Merging}

Local merging is a newly proposed stage by us to merge adjacent regions locally for drastically reducing the number of regions to be used at the stage of global merging. The SGF of each $4 \times 4$ subblock are obtained by averaging the texture features of all pixels within the subblock, so does the $L^{\star} a^{\star} b^{\star}$ color features of each subblock. 
The homogeneity between any current region and its neighboring adjacent region is measured individually. Then the two adjacent regions having the largest homogeneity measure $H_{\max }$ are regarded as similar and merged to become one region if the value of $H_{\max }$ is higher than a threshold 0.5 . The process is continued until all regions are scanned. We set the threshold 0.5 to avoid over merging in this stage. The result of local merging is shown in Fig. 4(c).

\subsection{Global Merging}

Global merging is a stage to merge similar adjacent regions globally. A pair of adjacent regions with the smallest merger importance value among all possible mergers in the entire image will be merged at each step. Merger importance $M I$ is defined as the ratio of the number of pixels in the smaller region to its homogeneity measure of adjacent regions

$$
M I=\frac{P_{\text {small }}}{H} .
$$

The procedure finds the best possible pair of adjacent regions globally whose merging introduces the smallest change in the segmented image. Since global merging reduces the number of segmented regions one by one at each step and it removes unimportant regions first, the essential regions remain to the end and thus perceptual segmentation is achieved.

It is also easy to stop the algorithm when the number of segmented regions reaches the specified number of segmented regions $N$. Fig. $4(d)$ shows the result of global merging when we set $N=6$ to obtain perceptual segmentation.

\subsection{Boundary Refinement}

Boundary refinement is finally performed to improve the localization of boundaries. If an image pixel is on the boundary of at least two distinct regions, a discrete disk with radius 3 will be placed on it. Then the homogeneity measure $H$ between the disk and its neighboring region is calculated individually to decide if the pixel needs to be relabeled. The next scan will check the neighborhoods of the relabeled pixels until no pixels are relabeled. The final segmentation after boundary refinement is shown in Fig. 4(e).

In the practical implementation, the user can easily choose the desirable optimal result with an appropriate roughness from among the several-segmented results. The results of segmentation when $N=8,7,6$ and 5 are shown clockwise from the upper left in Fig. $4(f)$. This figure demonstrates how the number of segmented regions decreases in the proposed algorithm.

\section{Experimental Results}

In this section, we present experimental results to assess the performance of the proposed segmentation method. For comparison, we show the results by 


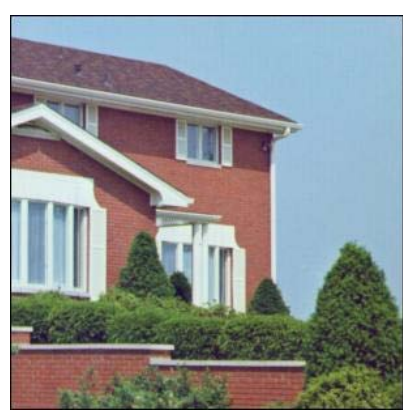

(a)

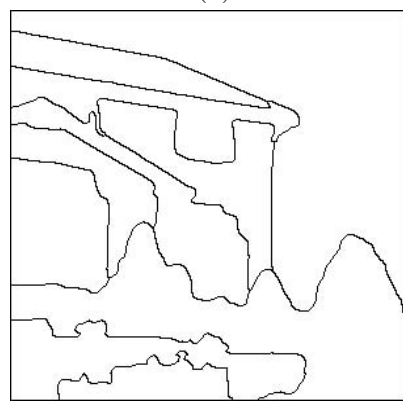

(c)

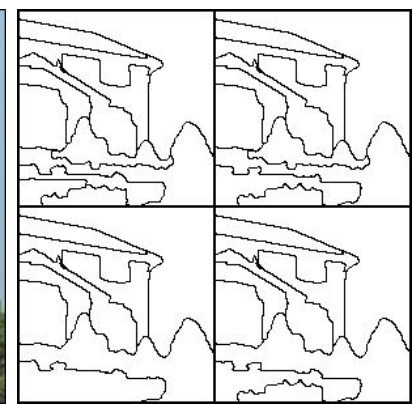

(b)

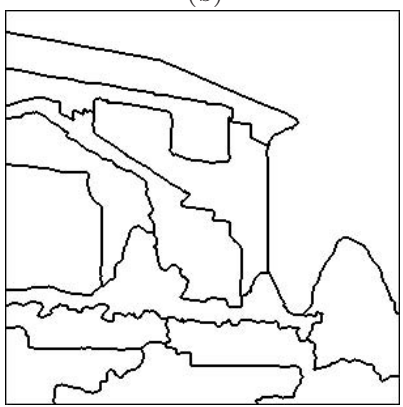

(d)

Fig. 5. Segmentation of a natural color image containing man-made objects: (a) original image; (b) results of segmentation with $N=12,11,10$ and 9 shown clockwise from the upper left; (c) result by proposed method with $N=10$; (d) result by EdgeFlow method

the EdgeFlow method using the algorithm made by the authors [15. Since the EdgeFlow algorithm cannot produce the specified number of segmented regions, we chose the best result with nearest number of segmented regions to our result by tuning the parameters. Since we set $\Delta S_{\text {small }}=0$ and $\Delta L_{\text {small }}=0$ in Fig. 2 , we have to tune two parameters $\Delta S_{\text {large }}$ and $\Delta L_{\text {large }}$ of the fuzzy membership functions differently according to each image and they were decided empirically.

We apply the proposed method to a $300 \times 300$ natural color image containing man-made objects shown in Fig. 5(a). The image is composed of the sky, a house, trees and a wall. The boundaries within the house further divide it into the main parts of objects such as roofs and windows. The perceptual segmentation is rather difficult because it is necessary to obtain accurate boundaries as well as uniform texture regions. Fig. $5(b)$ is the results of perceptual segmentation by the proposed algorithm when $N=12,11,10$ and 9 . The user can easily decide the optimal result by observing these segmented results. The selected optimal result with $N=10$ is shown in Fig. $5(c)$ and the result by the EdgeFlow method is shown in Fig. $5(d)$, respectively. We only show the boundaries of the segmented regions for clarity. Although two algorithms show the same degree of rough segmentation, the proposed algorithm represents a slightly better result than 


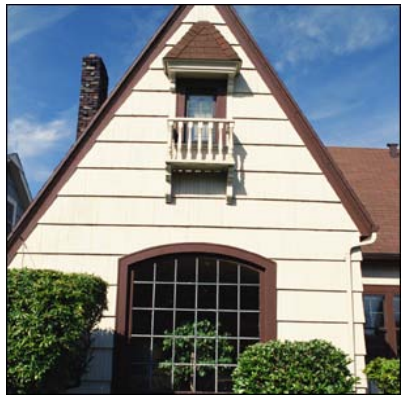

(a)

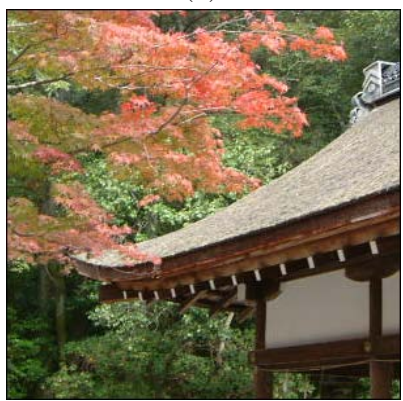

(d)

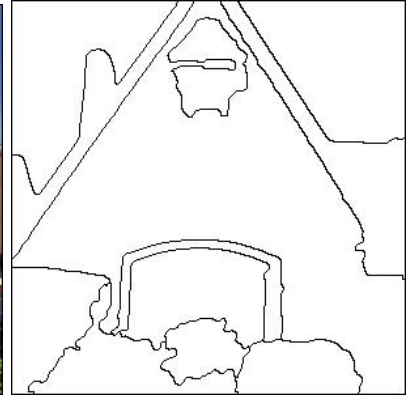

(b)

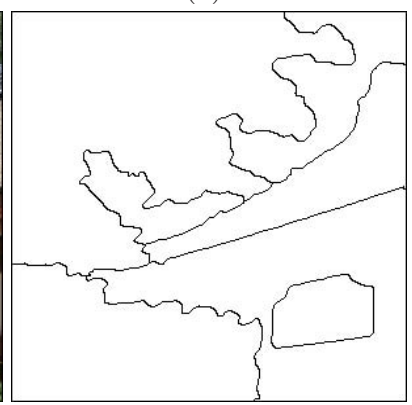

(e)

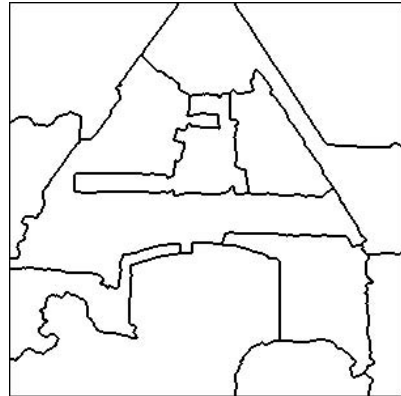

(c)

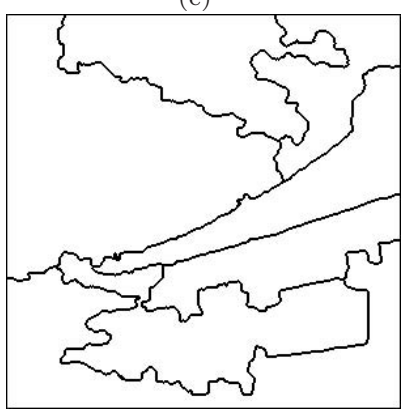

(f)

Fig. 6. Segmentation of a natural color image containing man-made objects: (a) and (d) original images; (b) and (e) results by proposed method with $N=12$ and $N=7$; (c) and (f) results by EdgeFlow method

the EdgeFlow method. In Fig. 5(c), the trees and the main parts of the house are well maintained as complete regions, and thus the segmented regions are more uniform and the boundaries of each region are maintained spatially more accurate than the result by the EdgeFlow method. The comparison demonstrates the effectiveness of the proposed method.

We then apply the proposed method to $300 \times 300$ natural color images shown in Fig. $6(a)$ and $(d)$. The results of perceptual segmentation by the proposed algorithm when $N=12$ and $N=7$ are shown in Fig. $6(b)$ and $(e)$, respectively. The results of segmentation by the EdgeFlow method are shown in Fig. 6(c) and $(f)$. These results also represent that the proposed algorithm produces perceptual segmentation more accurately than the EdgeFlow method. However, further investigations are necessary to precisely compare the proposed algorithm with other methods.

In order to assess the time cost by the newly introduced local merging stage, we compared the processing time with and without this stage. As a result, the processing time with this stage became $12-3 \%$ of the time without this stage. Thus, the introduction of local merging was confirmed to be effective to reduce the total computational cost. 


\section{Conclusions}

In this paper, we presented unsupervised perceptual segmentation of natural color images using the proposed fuzzy-based hierarchical algorithm that makes a reliable fusion of the $L^{\star} a^{\star} b^{\star}$ color features and the SGF texture features. The hierarchical segmentation using the fuzzy-based homogeneity measure is effective in obtaining perceptual segmentation that maintains uniform texture regions and accurate boundaries. The proposed algorithm has the prospective advantage of the capability to determine the desirable optimal result with an appropriate roughness, since it can produce the segmentation results by reducing the number of segmented regions one by one at each step.

\section{References}

1. Fu, K.S., Mu, J.K.: A survey on image segmentation. Pattern Recognition 13(1), 3-16 (1981)

2. Haralick, R.M., Shapiro, L.G.: Image segmentation techniques. Comput. Vision Graphics Image Processing 29, 100-132 (1985)

3. Pal, N.R., Pal, S.K.: A review on image segmentation techniques. Pattern Recognition 26(9), 1277-1294 (1993)

4. Reed, T.R., du Buf, J.M.H.: A review of recent texture segmentation and feature extraction techniques. CVGIP: Image Understanding 57, 359-372 (1993)

5. Mirmehdi, M., Petrou, M.: Segmentation of color textures. IEEE Trans. Pattern Anal. Mach. Intell. 22(2), 142-159 (2000)

6. Shi, J., Malik, J.: Normalized cuts and image segmentation. IEEE Trans. Pattern Anal. Mach. Intell. 22(8), 888-905 (2000)

7. Ma, W.Y., Manjunath, B.S.: EdgeFlow: A technique for boundary detection and image segmentation. IEEE Trans. Image Processing 9(8), 1375-1388 (2000)

8. Chen, J., Pappas, T.N., Mojsilovic, A., Rogowitz, B.E.: Adaptive perceptual colortexture image segmentation. IEEE Trans. Image Processing 14(10), 1524-1536 (2005)

9. Maeda, J., Novianto, S., Saga, S., Suzuki, Y., Anh, V.V.: Rough and accurate segmentation of natural images using fuzzy region-growing algorithm. In: Proc. IEEE Int. Conf. on Image Processing, vol. 3, pp. 227-231 (1999)

10. Maeda, J., Ishikawa, C., Novianto, S., Tadehara, N., Suzuki, Y.: Rough and accurate segmentation of natural color images using fuzzy region-growing algorithm. In: Proc. 15th Int. Conf. on Pattern Recognition, vol. 3, pp. 642-645 (2000)

11. Novianto, S., Suzuki, Y., Maeda, J.: Near optimum estimation of local fractal dimension for image segmentation. Pattern Recognition Letters 24(1-3), 365-374 (2003)

12. Ojala, T., Pietikäinen, M.: Unsupervised texture segmentation using feature distributions. Pattern Recognition 32(3), 477-486 (1999)

13. Chen, Y.Q., Nixon, M.S., Thomas, D.W.: Statistical geometrical features for texture classification. Pattern Recognition 28(4), 537-552 (1995)

14. Zadeh, L.A.: Fuzzy sets. Inform. Control 8, 338-353 (1965)

15. http://vision.ece.ucsb.edu/segmentation/edgeflow/software/ 